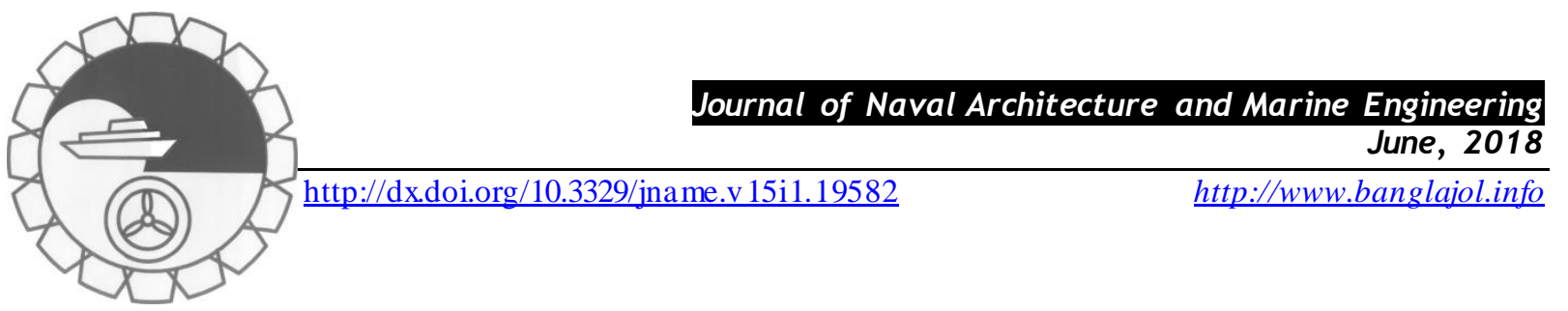

\title{
INTERNAL HEAT GENERATION EFFECT ON RADIATION HEAT TRANSFER MHD DISSIPATING FLOW OF A MICROPOLAR FLUID WITH VARIABLE WALL HEAT FLUX
}

\section{P. Sreenivasulu ${ }^{1}$, T. Poornima ${ }^{2}$ and N. Bhaskar Reddy ${ }^{3}$}

${ }^{1}$ Department of Mathematics, S.V. College of Engineering for Women, Tirupati-517502, A.P.,psreddysvu11@gmail.com

${ }^{2}$ School of Advanced Sciences (Mathematics), VIT University, Vellore- 632014.T.N., poornima.t@ vit.ac.in

${ }^{3}$ Department of Mathematics, Sri Venkateswara University, Tirupati- 517502.A.P., nbrsvu@ gamil.com

\begin{abstract}
:
An investigation is made to analyze the effects of heat generation/absorption and viscous dissipation on an unsteady MHD mixed convection flow of a viscous incompressible fluid past a vertical porous plate, in the presence of variable wall heat flux. Hence the governing boundary layer equations of the flow field are converted to into a system of non-linear ordinary differential equations by perturbation technique and then solved employing Runge-Kutta method with shooting technique. The effects of the various parameters on the translational and angular (micro-rotation) velocity, and temperature as well as the skin friction coefficient and couple stress coefficient at the surface are computed and discussed in detail with various values of the fluid properties. The numerical results of the local skinfriction coefficient and wall couple stress are given in a tabular form and discussed. Presence of micro particles in the fluid induces more heat into the fluid, thus raising the fluid temperature with the heat generation and also the viscous dissipation. The present results are compared with the existing literature and have a good agreement.
\end{abstract}

Keywords: Unsteady flow, thermal radiation, MHD, convection, micropolar fluid, viscous dissipation.

\begin{tabular}{|c|c|c|c|}
\hline \multicolumn{2}{|c|}{ NOMENCLATURE } & $C p$ & specific heat at constant pressure \\
\hline $\mathrm{u}^{*}, \mathrm{v}^{*}$ & velocity components & \multicolumn{2}{|c|}{ Greek symbols } \\
\hline$u, v$ & dimensionless velocity components & $\beta$ & coefficient of thermal expansion \\
\hline$T$ & the temperature of the fluid & $v$ & kinematic viscosity \\
\hline$T_{\infty}$ & Ambient temperature & $\psi$ & stream function \\
\hline $\mathrm{K}^{*}$ & the permeability of the porous medium & $\theta$ & dimensionless temperature \\
\hline$q_{r}$ & the radiative heat flux & $\rho$ & density \\
\hline$p^{*}$ & the pressure & $\varepsilon$ & small parameter such that $\varepsilon B \leq 1$ \\
\hline$U_{0}$ & scale of free steam velocity & $B_{0}$ & magnetic induction \\
\hline$B, n^{*}, A$ & real positive constants & $\sigma$ & electric conductivity \\
\hline$V_{0}$ & scale of suction velocity $(>0)$. & $\beta_{f}$ & $\begin{array}{l}\text { thermal expansion coefficients } \\
\text { fluid }\end{array}$ \\
\hline$G r$ & thermal Grashof number & $\alpha$ & fluid thermal diffusivity \\
\hline $\operatorname{Pr}$ & Prandtl Number & $v_{r}$ & fluid kinematic rotational viscosity \\
\hline$R, E c$ & radiation parameter,Eckert number & $g$ & the acceleration due to gravity \\
\hline$M$ & magnetic field parameter & $\sigma_{s}$ & Stefan-Boltzmann constant \\
\hline$Q$ & the heat generation parameter & $\omega^{*}$ & the component of the angular velocity \\
\hline$j^{*}$ & micro-inertia density & $\Gamma$ & the spin-gradient viscosity \\
\hline$k_{e}$ & the mean absorption coefficient & $\Lambda$ & coefficient of gyro-viscosity \\
\hline
\end{tabular}




\section{Introduction}

Micropolar fluids are fluids with microstructure belonging to the class of fluids with non-symmetric stress tensor called as polar fluids which includes the special case, the classical fluids Navier-Stokes fluid model usually referred as ordinary fluids. There are a large number of fluids existing in nature that contain suspension of small particles. The properties of such type of fluids are different from those of Newtonian fluid due to fluid particle interaction and rotation. The minuscule effects arising from the local structure and micro-motions of the fluid elements have been taken into account in the theory of micropolar fluid, which was first introduced and formulated by Eringen $(1996,1972)$. His theory of micropolar fluids has opened up new areas in research in the physics of fluid flow. According to him, a simple micro fluid is a fluent medium whose properties and behaviour are affected by the local motions of the material particles contains in each of its volume elements, such a fluid possesses local inertia. Physically, they represent the fluids consisting of randomly oriented particles suspended in a viscous medium. Synovial fluid is a good example of micropolar fluids. The theory is expected to provide a mathematical model, which can be used to describe the behavior of non-Newtonian fluids such as polymeric fluids, liquid crystals, paints, animal blood, colloidal fluids, Ferro-liquids, etc., for which the classical Navier-Stokes theory is inadequate. Eringen (1996) proposed the theory of micropolar fluids which show microrotation effects as well as micro-inertia. Later on, theory of thermos-micropolar fluids was developed by Eringen (1972) by taking thermal effects into account. A comprehensive review of micropolar fluids theory was presented by Ariman et al. (1974). Jena and Mathur (1981) studied the laminar free convection in the boundary layer flow of the thermo-micropolar fluids past a non-isothermal vertical plate. Sharma and Gupta (1995) considered thermal convection in micropolar fluids in porous medium.

The study of flow and heat transfer for an electrically conducting fluid past a porous plate has attracted the interest of many investigators in view of its applications in many engineering problems such as oil exploration, geothermal energy extractions and the boundary layer control in aerodynamics (Soundalgekar, 1973, Kim, 2000, and Kim, 2001a). Specifically, Soundalgekar (1973) obtained approximate solutions for the two dimensional flow of an incompressible, viscous fluid flow past an infinite porous vertical plate with constant suction velocity normal to the plate. He found that the difference between the temperature of the plate and the free stream is significant to cause the free convection currents.

The study of MHD phenomena is characterized by a mutual interaction between the fluid velocity field (hydrodynamic boundary layer) and the electromagnetic field. In recent years, the subject of MHD has attracted the attention of many authors, due to many applications to problems of eng ineering and industrial nature such as MHD power generators and accelerators, geothermal energy extractions and crystal growth. Kim (2001b) studied unsteady MHD convection flow of polar fluids past a semi-infinite vertical-moving porous plate in a porous medium. El-Hakiem et al. (1999) studied the effect of viscous and Joule heating on MHD-free convection flow with variable plate temperature in a micropolar fluid using the Keller-box implicit scheme. Rahman and Sattar (2006) analyzed magnetohydrodynamic convective flow of a micropolar fluid past a continuously moving vertical porous plate in the presence of heat generation/absorption. Similarity transformations of heat and mass transfer effects on steady MHD free convection dissipative fluid flow past an inclined porous surface with chemical reaction was investigated by Reddy et al. (2014). Sultana et al. (2011) studied the micropolar fluid behavior on MHD heat transfer flow through a porous medium with induced magnetic field by finite difference method. Patowary (2012) investigated the effect of variable viscosity and thermal conductivity of micropolar fluid in a porous channel in presence of magnetic field. Sreenivasulu et al. (2014) analyzed the variable suction effect on Magnetohydrodynamic convective and radiating flow past a vertical porous moving surface.

On the other hand, heat transfer by simultaneous free or mixed convection and thermal radiation in the case of a micropolar fluid has not received as much attention. This is unfortunate because thermal radiation plays an important role on determining the overall surface heat transfer in situations where convective heat transfer coefficients are small. Such situations are common in space technology (Soundalgekar,1977). Raptis (1998) studied numerically the case of a steady two-dimensional flow of a micropolar fluid past a continuously moving plate with a constant velocity in the presence of thermal radiation. Gorla and Tornabene (1988) investigated the effects of thermal radiation on mixed convection flow over a vertical plate with non-uniform heat flux boundary conditions. Mustafa et al. (2012) analyzed the MHD stagnation point flow of a micropolar fluid towards a moving surface with radiation. Rahman and Sultana (2008) studied the radiative heat transfer flow of micropolar fluid with variable heat flux in a porous medium. Poornima et al.(2014), studied the slip flow of Casson rheological fluid under variable thermal conductivity with radiation effects. Reddy (2016), presented the MHD 
boundary layer slip flow of a Casson fluid over an exponentially stretching surface in the presence of thermal radiation and chemical reaction.

As the flow is under high gravitation or the field of the flow may be extreme size, in such case, it is necessary to study the effect of viscous dissipation. Viscous dissipation is a term which is always positive and represents a source of heat due to friction between the fluid particles. But viscous dissipation in the natural convection flow is important, when the flow field is of extreme size or in high gravitational field. Gebhart and Mollendorf (1969) considered the effects of viscous dissipation for external natural convection flow over a surface. Soundalgekar (1972) analyzed viscous dissipative heat on the two-dimensional unsteady free convective flow past an infinite vertical porous plate. Israel-Cookey et al. (2003) investigated the influence of viscous dissipation and radiation on unsteady MHD free convection flow past an infinite heated vertical plate in a porous medium with time dependent suction. Duwairi (2005) has presented the effects of Joule heating and viscous dissipation on the forced convection flow in the presence of thermal radiation. Ibrahim et al.( 2008) studied the Influence of viscous dissipation and radiation on unsteady MHD mixed convection flow of micropolar fluids. Ishak et al. (2006) presented the Flow of a micropolar fluid on a continuous moving surface in the presence of viscous dis sipation.

The study of heat generation or absorption in moving fluids is important in problems dealing with chemical reactions and those concerned with dissociating fluids. Possible heat generation effects may alter the temperature distribution consequently, the particle deposition rate in nuclear reactors, electronic chips and semiconductor wafers. Simple mathematical models are available as its exact modeling is not possible, where it exhibits its average behaviour. The volumetric heat generation rate $Q\left[\mathrm{~W} / \mathrm{m}^{3}\right]$ as s u mes the following by

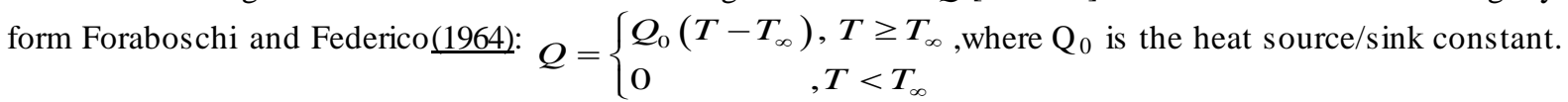

The above relation is valid for the state of some exothermic processes having $T_{\infty}$ as the ambient temperature. Many researchers worked on the effect of it. Khedr et al. (2009) studied the effects of suction/ injection and heat generation on MHD flow of a micropolar fluid past a stretched permeable surface. Mohamed et al. (2011) analyzed the Heat and mass transfer analysis on the flow of non-Newtonian micropolar fluid with uniform suction/blowing, heat generation, chemical reaction and thermophoresis effects. Abdel-Rahman et al. (2011) investigated the Heat transfer over an unsteady moving surface with heat generation and thermal radiation in micropolar fluid in the presence of suction/injection. Reddy (2012) presented the magnetohydrodynamics and radiation effects on unsteady convection flow of micropolar fluid past a vertical porous plate with variable wall heat flux by employing traditional analytical perturbation method. Okedoye, (2014) studied the unsteady MHD mixed convection flow past an oscillating plate with heat source/sink. Sreenivasulu et al. (2017) analyzed the variable thermal conductivity influence on Hydromagnetic flow past a stretching cylinder in a thermally stratified medium with heat source/sink.

In this paper an attempt is made to study the effects of thermal radiation and viscous dissipation effects on unsteady MHD mixed convection flow of micropolar fluid past a vertical porous plate with variable wall heat

\section{Mathematical Analysis}

Consider an unsteady, two dimensional mixed convection boundary layer flow of a viscous incompressible electrically conducting and radiating micropolar fluid past an infinite vertical porous plate with variable wall heat flux and heat generation/absorption. The $x^{*}$-axis is taken along the vertical porous plate in an upward direction and $y^{*}$-axis is taken normal to the plate. The transversely applied magnetic field and magnetic Reynolds number are very small and hence the induced magnetic field is negligible. Viscous and Darcy resistance terms are taken into account as there is constant permeability porous medium. It is also assumed that the free stream to consist of a mean velocity and temperature over which are superimposed an exponentially varying with time. Under the usual Boussinesq's approximation, the equation of continuity, linear momentum, micro-rotation, and energy can be written as:

$$
\begin{aligned}
& \frac{\partial v^{*}}{\partial y^{*}}=0 \\
& \frac{\partial u^{*}}{\partial t^{*}}+v^{*} \frac{\partial u^{*}}{\partial y^{*}}=-\frac{1}{\rho} \frac{\partial p^{*}}{\partial x^{*}}+\left(v+v_{r}\right) \frac{\partial^{2} u^{*}}{\partial y^{*}}+g \beta_{f}\left(T-T_{\infty}\right)-\frac{v}{K} u^{*}-\frac{\sigma B_{0}^{2}}{\rho} u^{*}+2 v_{r} \frac{\partial \omega^{*}}{\partial y^{*}}
\end{aligned}
$$




$$
\begin{aligned}
& \rho j *\left(\frac{\partial \omega^{*}}{\partial t^{*}}+v^{*} \frac{\partial \omega^{*}}{\partial y^{*}}\right)=\gamma \frac{\partial^{2} \omega^{*}}{\partial *^{* 2}} \\
& \frac{\partial T}{\partial t^{*}}+v^{*} \frac{\partial T}{\partial y^{*}}=\alpha \frac{\partial^{2} T}{\partial y^{2}}-\frac{1}{\rho C_{p}} \frac{\partial q_{r}}{\partial y^{*}}+\frac{\mu}{\rho C_{p}}\left(\frac{\partial u^{*}}{\partial y^{*}}\right)^{2}+\frac{Q_{0}}{\rho C_{p}}\left(T-T_{\infty}\right)
\end{aligned}
$$

The appropriate boundary conditions for the velocity, microrotation, and temperature fields are

$$
\begin{aligned}
& u^{*}=0, \quad \omega^{*}=-\frac{1}{2} \frac{\partial u^{*}}{\partial y^{*}}, \quad \frac{\partial T}{\partial y^{*}}=-\frac{q_{w}}{k}\left(1+\varepsilon B e^{n^{*} t^{*}}\right) \quad \text { at } \quad y^{*}=0 \\
& u^{*} \rightarrow U^{*}=U_{0}\left(1+\varepsilon e^{n^{* *^{*}}}\right) \quad \text { at } \omega^{*} \rightarrow 0, \\
& T \rightarrow T_{\infty}, \quad \text { as } y^{*} \rightarrow \infty
\end{aligned}
$$

The radiative heat flux $q_{r}$ is described by Rosseland approximation, such that

$q_{r}=-\frac{4}{3} \frac{\sigma_{s}}{k_{e}} \frac{\partial T^{4}}{\partial \bar{y}}$

It should be noted that by Rosseland approximation, we limit our analysis to optically thick fluids. If the temperature differences within in the flow are sufficiently small, then Equation (4) can be linearized by expanding $T^{4}$ into the Taylor series about $T_{\infty}$ and neglecting higher order terms to take the form: $T^{4} \cong 4 T_{\infty}^{3} T-3 T_{\infty}^{4}$

In view of Equations (6) and (7), equation (4) becomes

$$
\frac{\partial T}{\partial t^{*}}+v^{*} \frac{\partial T}{\partial y^{*}}=\alpha\left(1+\frac{16 \sigma_{s} T_{\infty}^{3} V_{0}^{2}}{3 k k_{e} v^{2}}\right) \frac{\partial^{2} T}{\partial y^{* 2}}+\frac{\mu}{\rho C_{p}}\left(\frac{\partial u^{*}}{\partial y^{*}}\right)^{2}+\frac{Q_{0}}{\rho C_{p}}\left(T-T_{\infty}\right)
$$

From Equation (1), it is clear that the suction velocity at the plate is either a constant or a function of time. Hence the suction velocity normal to the plate is assumed in the form

$$
v^{*}=-V_{0}\left(1+\varepsilon A e^{n^{* * *}}\right)
$$

The negative sign indicates that the suction is towards the plate. Outside the boundary layer, Equation (2) gives

$$
-\frac{1}{\rho} \frac{d p^{*}}{d x^{*}}=\frac{d U_{\infty}^{*}}{d t^{*}}+\frac{v}{K^{*}} U_{\infty}^{*}+\frac{\sigma B_{0}^{2}}{\rho} U_{\infty}^{*}
$$

Introducing the following non-dimensional quantities

$$
\begin{aligned}
& u=\frac{u^{*}}{U_{0}}, v=\frac{v^{*}}{U_{0}}, y=\frac{V_{0} y^{*}}{v}, t=\frac{V_{0}^{2} t^{*}}{v}, U_{\infty}=\frac{U_{\infty}^{*}}{U_{0}}, \theta=\frac{\left(T-T_{\infty}\right) k V_{0}}{\left(T_{w}-T_{\infty}\right) q_{w}}, \omega=\frac{v}{U_{0} V_{0}} \omega^{*}, n=\frac{v n^{*}}{V_{0}^{2}}, \\
& K=\frac{K^{*} V_{0}^{2}}{v^{2}}, \operatorname{Pr}=\frac{v}{\alpha}, M=\frac{\sigma B_{0}^{2} v}{\rho V_{0}^{2}}, R=\frac{4 \sigma_{s} T_{\infty}^{3}}{k_{e} k}, G r=\frac{g v \beta_{f}\left(T_{w}-T_{\infty}\right)}{U_{0} V_{0}^{2}}, E c=\frac{U_{0}^{2} V_{0} k}{q_{w} C p\left(T_{w}-T_{\infty}\right)}, \\
& \gamma=\left(\mu+\frac{\Lambda}{2}\right) j^{*}=\mu j^{*}\left(1+\frac{1}{2} \beta\right), Q=\frac{k Q_{0} v}{\rho C p V_{0} q_{w}}, \eta=\frac{\mu j^{*}}{\gamma}=\frac{2}{2+\beta}, \beta=\frac{\Lambda}{\mu}
\end{aligned}
$$

Equations (2), (3) and (8) take the following dimensionless form,

$$
\begin{aligned}
& \frac{\partial u}{\partial t}-\left(1+\varepsilon A e^{n t}\right) \frac{\partial u}{\partial y}=\frac{d U_{\infty}}{d t}+(1+\beta) \frac{\partial^{2} u}{\partial y^{2}}+G r \theta+N\left(U_{\infty}-u\right)+2 \beta \frac{\partial w}{\partial y} \\
& \frac{\partial \omega}{\partial t}-\left(1+\varepsilon A e^{n t}\right) \frac{\partial \omega}{\partial y}=\frac{1}{\eta} \frac{\partial^{2} \omega}{\partial y^{2}} \\
& \frac{\partial \theta}{\partial t}-\left(1+\varepsilon A e^{n t}\right) \frac{\partial \theta}{\partial y}=\frac{1}{\operatorname{Pr}}\left(1+\frac{4 R}{3}\right) \frac{\partial^{2} \theta}{\partial y^{2}}+E c\left(\frac{\partial u}{\partial y}\right)^{2}+Q \theta
\end{aligned}
$$

The corresponding boundary conditions are

$$
\begin{gathered}
u=0, \quad \frac{\partial \theta}{\partial y}=-\left(1+\varepsilon B e^{n t}\right), \quad \omega=-\frac{1}{2} \frac{\partial u}{\partial y} \quad \text { at } \quad y=0 \\
u \rightarrow U_{\infty}, \quad \theta \rightarrow 0, \quad \omega \rightarrow 0 \quad \text { as } \quad y \rightarrow \infty
\end{gathered}
$$




\section{Solution of the Problem}

Equations (12) - (14) are coupled, non-linear partial differential equations and these cannot be solved in closedform. However, these equations can be reduced to a set of ordinary differential equations, which can be solved analytically. This can be done by representing the velocity, micro-rotation and temperature of the fluid in the neighborhood of the porous plate as

$$
\begin{aligned}
& u=u_{0}(y)+\varepsilon e^{n t} u_{1}(y)+O\left(\varepsilon^{2}\right)+\ldots \\
& \omega=\omega_{0}(y)+\varepsilon e^{n t} \omega_{1}(y)+O\left(\varepsilon^{2}\right)+\ldots . \\
& \theta=\theta_{0}(y)+\varepsilon e^{n t} \theta_{1}(y)+O\left(\varepsilon^{2}\right)+\ldots
\end{aligned}
$$

Substituting Equation (16) in Equations (12) - (14) and equating the harmonic and non - harmonic terms, and neglecting the higher order terms of $O\left(\varepsilon^{2}\right)$, we obtain

Zero order terms of $\varepsilon$

$$
\begin{aligned}
& (1+\beta) u_{0}^{\prime \prime}+u_{0}^{\prime}+N\left(1-u_{0}\right)+G r \theta_{0}+2 \beta \omega_{0}^{\prime}=0 \\
& \omega_{0}^{\prime \prime}+\eta \omega_{0}^{\prime}=0 \\
& \left(1+\frac{4 R}{3}\right) \theta_{0}^{\prime \prime}+\operatorname{Pr} \theta_{0}^{\prime}+E c \operatorname{Pr} u_{0}^{\prime 2}+Q \operatorname{Pr} \theta_{0}=0
\end{aligned}
$$

The corresponding boundary conditions are

$$
\begin{aligned}
& u_{0}=0, \omega_{0}=-\frac{1}{2} u_{0}^{\prime}, \theta_{0}^{\prime}=-1 \quad \text { at } y=0 \\
& u_{0}=1, \quad \omega_{0} \rightarrow 0, \quad \theta_{0} \rightarrow 0 \quad \text { as } y \rightarrow \infty
\end{aligned}
$$

First order terms of $\varepsilon$

$$
\begin{aligned}
& (1+\beta) u_{1}^{\prime \prime}+u_{1}^{\prime}+(N+n)\left(1-u_{1}\right)+A u_{0}^{\prime}+G r \theta_{1}+2 \beta \omega_{1}^{\prime}=0 \\
& \omega_{1}^{\prime \prime}+\eta \omega_{1}^{\prime}-n \eta \omega_{1}+A \eta \omega_{0}^{\prime}=0 \\
& \left(1+\frac{4 R}{3}\right) \theta_{1}^{\prime \prime}+\operatorname{Pr} \theta_{1}^{\prime}+A \operatorname{Pr} \theta_{0}^{\prime}+(Q-n) \theta_{1}+2 E c u_{0}^{\prime} u_{1}^{\prime}=0
\end{aligned}
$$

The corresponding boundary conditions are

$$
\begin{aligned}
& u_{1}=0, \quad \omega_{1}=-\frac{1}{2} u_{1}^{\prime}, \theta_{1}^{\prime}=-B \quad \text { at } y=0 \\
& u_{1}=1, \quad \omega_{1} \rightarrow 0, \quad \theta_{1} \rightarrow 0 \quad \text { as } y \rightarrow \infty
\end{aligned}
$$

where prime denotes ordinary differentiation with respect to $y$.

But $\theta^{\prime}(0)=-1$, whenever heat flux is included, so B must be 0.1 as $\theta=\theta_{0}+x^{n t} \theta_{1}$.

The governing boundary layer equations (17) - (19) and (21) - (23) subject to the boundary conditions (20) and (24) are solved numerically using Runge-Kutta method with shooting technique.

From the technological point of view, the skin-friction, couple stresses are important physical quantities for this type of boundary layer flow.

The dimensionless form of the skin-friction coefficient, couple stress component and Nusselt number near the plate can be defined as,

$$
C_{f}=\frac{\tau_{w}^{*}}{\rho U_{0} V_{0}}=u^{\prime}(0) \text { where } \tau_{w}^{*}=\left.\mu \frac{\partial u *}{\partial y^{*}}\right|_{y^{*}=0}, C_{m}=\frac{M_{w}}{\mu j U_{0}}=\omega^{\prime}(0) \text { where } M_{w}=\left.\gamma \frac{\partial \omega^{*}}{\partial y^{*}}\right|_{y^{*}=0}
$$




\section{Results and Discussion}

A representative set of numerical results is shown graphically in Figs. 1 - 16, to illustrate the influence of physical parameters on the velocity, microrotation and temperature. In order to validate the present numerical results obtained by using the shooting technique, the present results are compared with that of Reddy (2012), for appropriate reduced cases, and found that there is an excellent agreement (see Table 1).

Table 1: Comparis on of $C_{f}$ and $C_{m}$ for different values of $G r, M, \operatorname{Pr}$ and $R$, when $E c=Q=0$.

\begin{tabular}{|c|c|c|c|c|c|c|c|}
\hline \multirow[b]{2}{*}{$G r$} & \multirow[b]{2}{*}{$M$} & \multirow[b]{2}{*}{$P r$} & \multirow[b]{2}{*}{$R$} & \multicolumn{2}{|l|}{$\overline{C_{f}}$} & \multicolumn{2}{|l|}{$C_{m}$} \\
\hline & & & & $\begin{array}{ll}\text { M G } & \text { Reddy } \\
(2012) & \end{array}$ & $\begin{array}{l}\text { Present } \\
\text { Sudy }\end{array}$ & $\begin{array}{ll}\text { M G } & \text { Reddy } \\
(2012) & \end{array}$ & $\begin{array}{l}\text { Present } \\
\text { Study }\end{array}$ \\
\hline 2.0 & 2.0 & 0.71 & 2.0 & 6.9567 & 6.95674 & 3.3146 & 3.31465 \\
\hline 4.0 & & & & 10.5384 & 10.53843 & 5.0936 & 5.09361 \\
\hline 2.0 & 3.0 & & & 6.7482 & 6.74821 & 3.1875 & 3.18754 \\
\hline & 2.0 & 2.0 & & 4.5211 & 4.52112 & 2.1690 & 2.16902 \\
\hline & & 0.71 & 4.0 & 6.1079 & 6.10795 & 2.9172 & 2.91723 \\
\hline
\end{tabular}

Figs. 1 and 2 exhibit the effect of magnetic field on translational and angular velocity. The effect of magnetic field is opposite to that of material property of the fluid with an exception i.e. transition layers almost coincide for two flows. To be clear, as the magnetic field generates a force of electromagnetic origin, this is a resistive force, so that the translational velocity decreases. From Fig. 2, it is also evident that an increase in magnetic field strength enhances the angular velocity at all points. The resistive force has contributed to enhance the velocity which may be attributed to the interplay of the effects of buoyancy force, heat source and viscous dissipation.

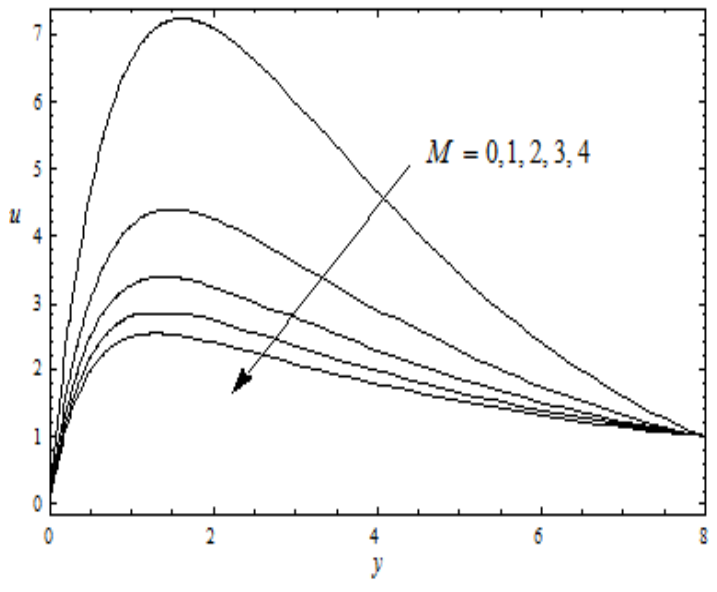

Fig. 1: Velocity profiles for different values of $M$

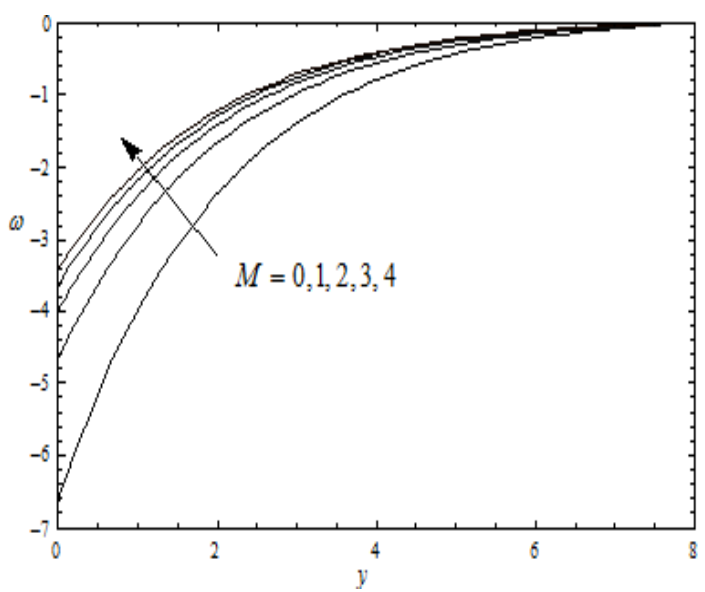

Fig. 2: Angular velocity profiles for different values of $M$

For different values of the thermal Grashof number $G r$, the translation velocity and micro-rotation are shown in Figs. 3 and 4. It is observed that an increase in $G r$ leads to a rise in the velocity profiles as the Grashof number is the ratio of buoyancy to viscous force, but decreases due to micro-rotation. In addition, the curves show that the peak value of velocity increases rapidly near the plate and then decays to the free stream velocity 


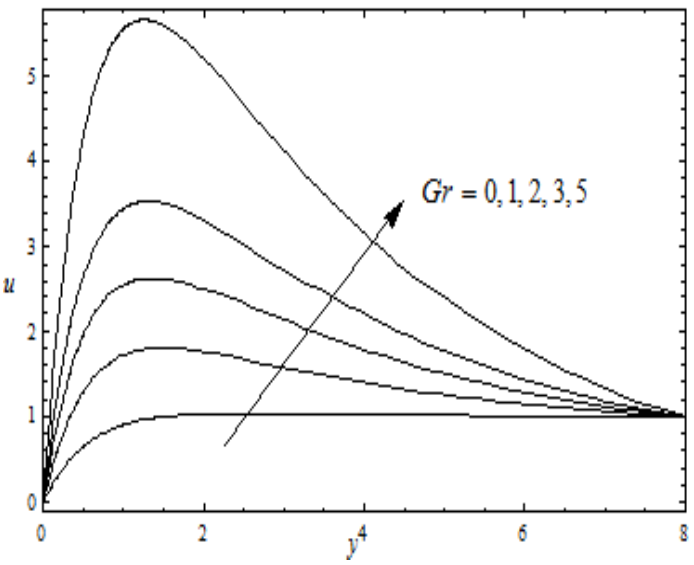

Fig. 3: Velocity profiles for different values of $\mathrm{Gr}$

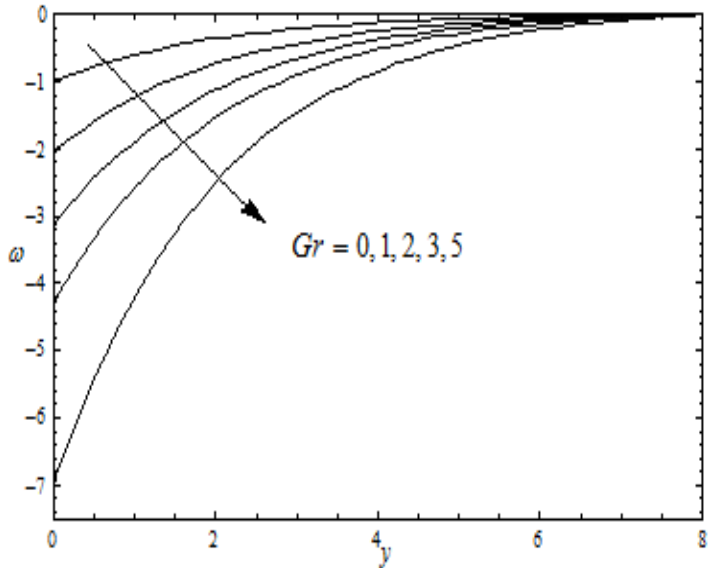

Fig. 4: Angular velocity profiles for different values of $\mathrm{Gr}$

Figs. 5 and 6 portray the effect of porosity parameter $K$ on the translational and angular velocity, respectively. It is obvious that the effect of increasing values of $K$ results in increasing velocity distribution across the boundary layer (Fig.5). The micro-rotation decreases as the permeability parameter increases (Fig.6). It is seen that the angular velocity profiles rises near the porous plate and get saturated or converges to the point zero.

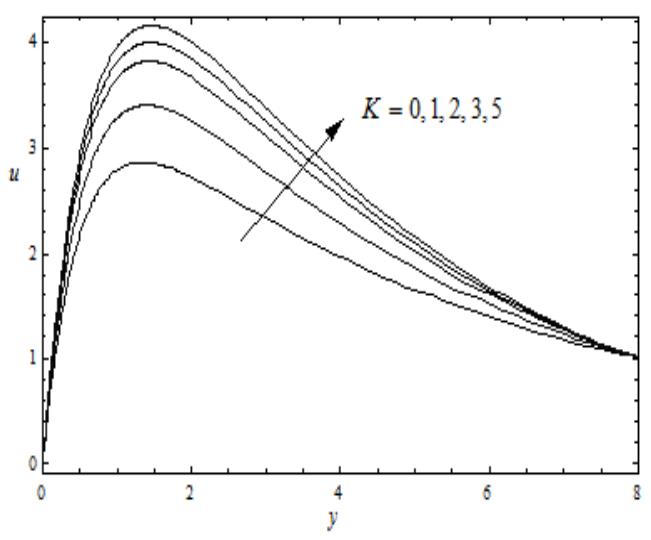

Fig. 5: Velocity profiles for different values of $K$

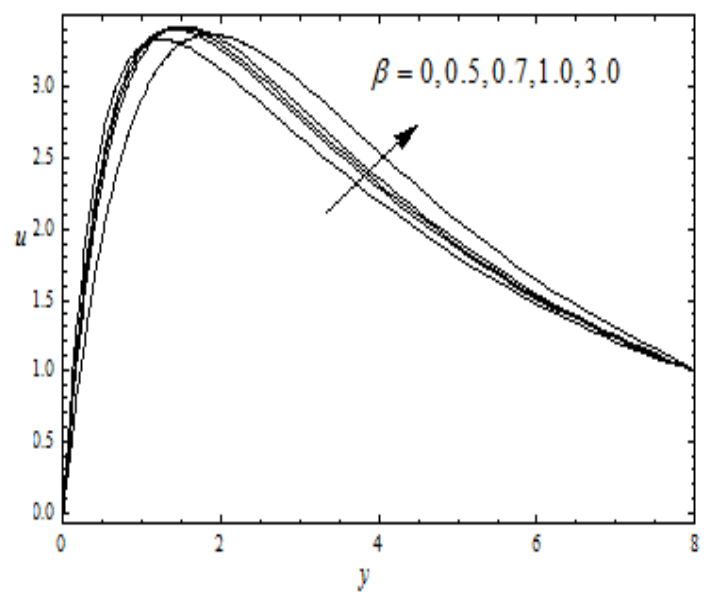

Fig. 7: Velocity profiles for different values of $\beta$

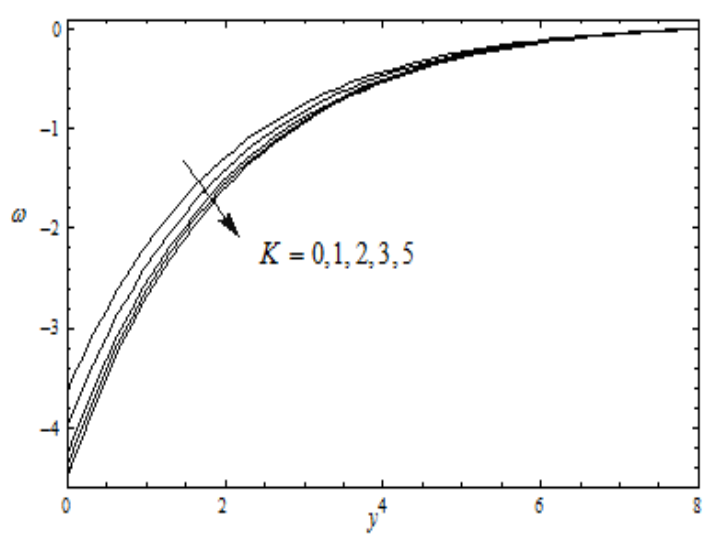

Fig. 6: Angular velocity profiles for different values of

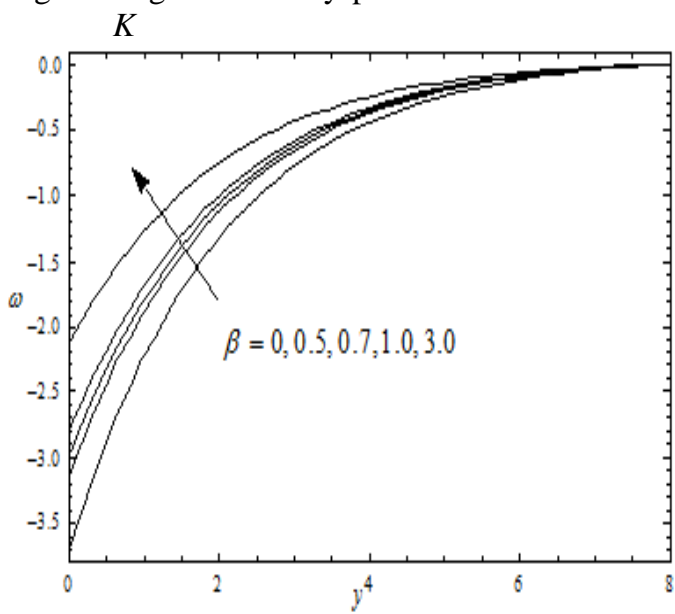

Fig. 8: Angular velocity profiles for different values of $\beta$

Fig. 8 presents the angular velocity variation in response to an increasing non-Newtonian property of the fluid present in the under study. It is seen that micro-rotation remains negative near the boundary layer at one point where profiles intersect at $y=6$. This indicates that these layers present transition state after which the opposite 
effect i.e. $|\omega|$ increases with an increasing $\beta$ till the free stream state is attained $(y>8.0)$. It is clear that the translation velocity increases near the plate reaches a maximum at $y=2$ and from there the profiles decreases merges with the free stream velocity. From Fig.9, it is seen that the effect of increasing values Pr on decreasing the translational velocity at the wall and then approach to the free stream boundary layer conditions.

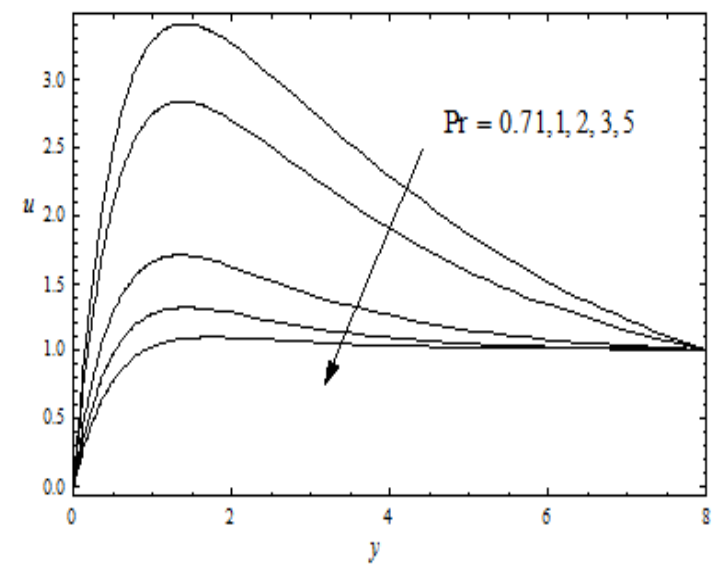

Fig. 9: Velocity profiles for different values of $P r$

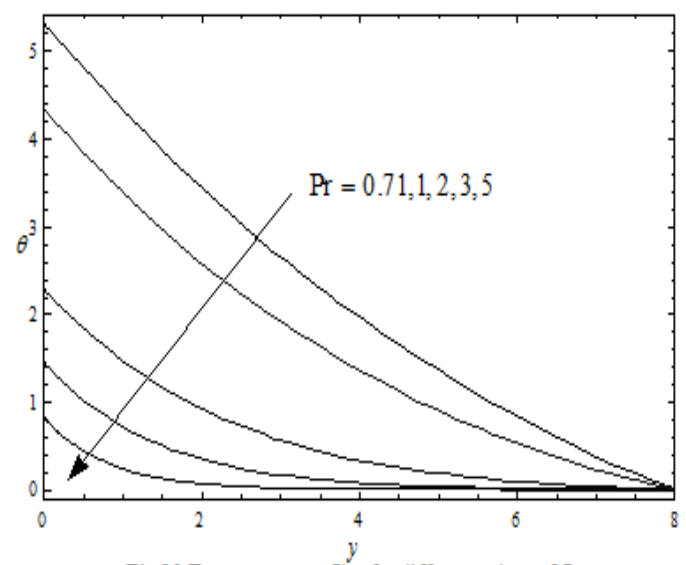

Fig. 10: Temperature profiles for different values of $\mathrm{Pr}$

The smaller values of $\operatorname{Pr}$ are equivalent to increasing the thermal conductivities, and therefore heat is able to diffuse away from the heated surface more rapidly than for higher values of $P r$. Hence, the boundary layer is thicker rising the fluid temperature (Fig.10) and rate of heat transfer is reduced, as gradients have been reduced. It is clear from Figs.11-12 that an increase in the radiation parameter results in increasing both the velocity and temperature within the boundary layer

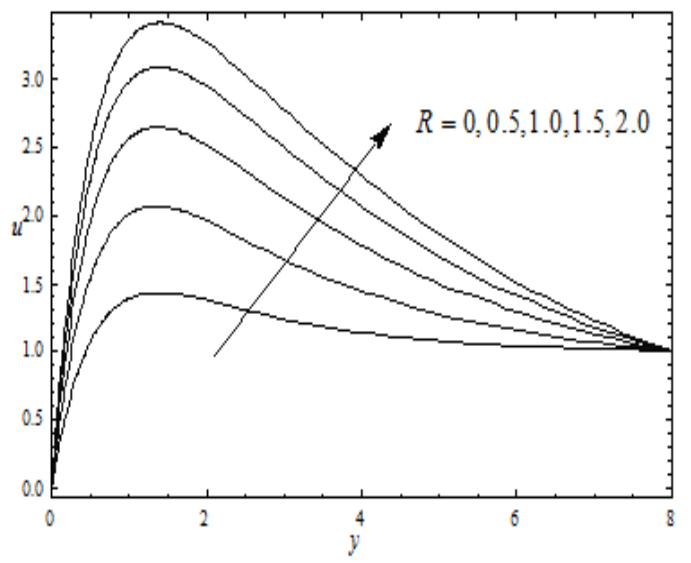

Fig. 11: Velocity profiles for different values of $R$

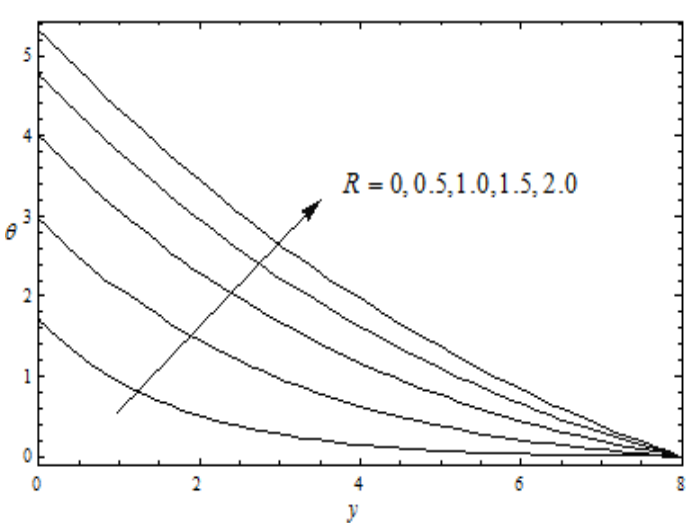

Fig. 12: Temperature profiles for different values of $R$

For different values of Eckert number on the translation velocity and temperature are depicted in Figs.13-14. Ec embodies the conservation of kinetic energy into internal energy by work done against the viscous fluid stress. The positive Eckert number implies cooling of the sheet i.e., loss of heat from the sheet to the fluid. It is found that the translation velocity and temperature as well as thermal boundary layer thickness increase slightly with an increase in $E c$.

Figs. 15 and 16 show the translation velocity and temperature for different values of heat generation/absorption parameter $Q$. Positive values of $Q$ represent the heat generation to the fluid and the negative values of $Q$ represent the heat absorption from the fluid. From Fig. 15, it is observed that when the heat is generated $(Q>0)$ the buoyancy force increases, which induce the flow rate to increase giving, rise to the increase in the translation velocity. Again when the heat absorption $(Q<0)$ intensifies the translation velocity is found to decrease due to the decrease in the buoyancy force. From Fig. 16, it is observed that the temperature increases as $Q$ increases. 


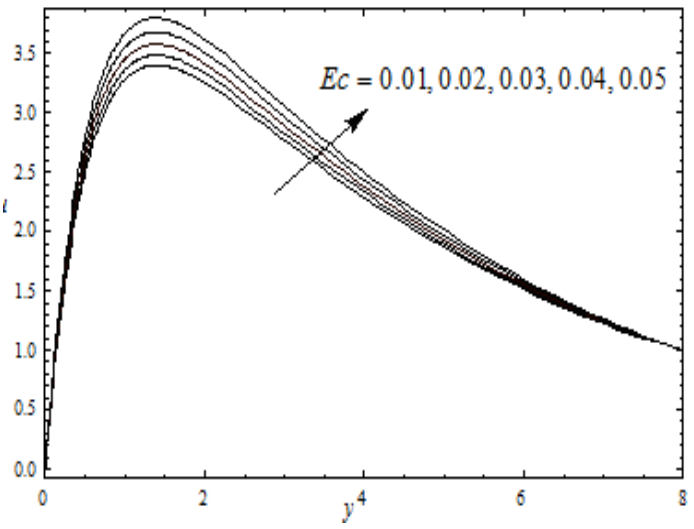

Fig. 13: Velocity profiles for different values of $E c$

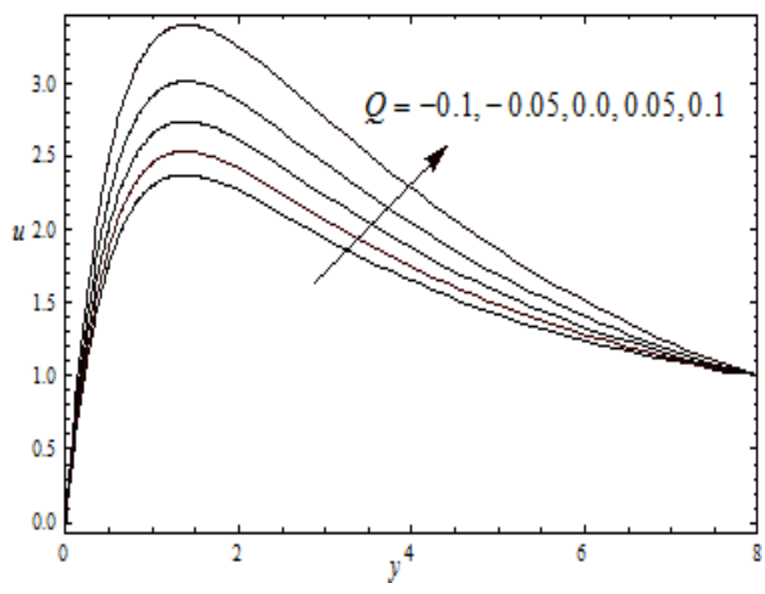

Fig. 15: Velocity profiles for different values of $Q$

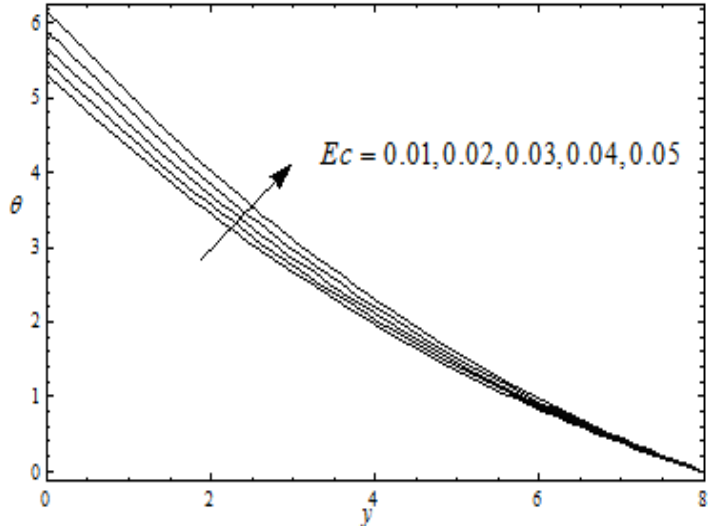

Fig. 14: Temperature profiles for different values of $E c$

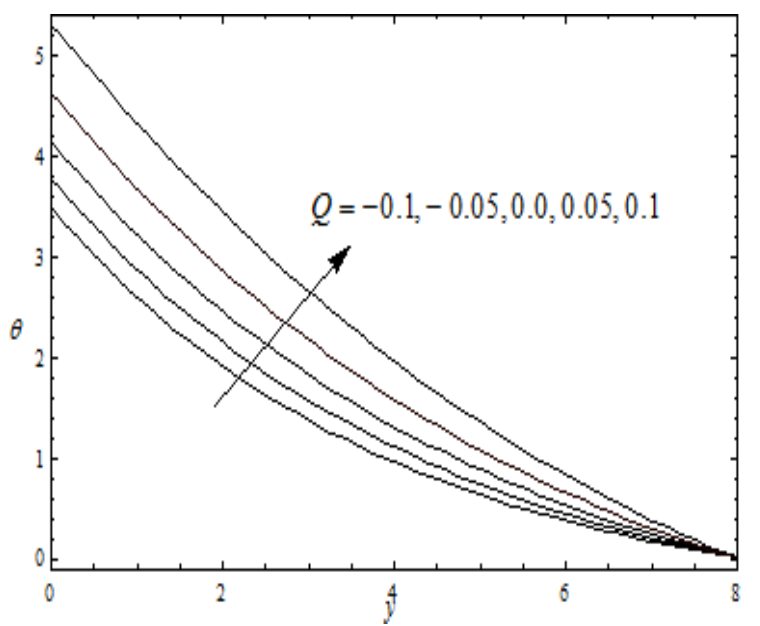

Fig. 16: Temperature profiles for different values of $Q$

Numerical values for functions proportional to shear stress and wall couple stress are presented in Table 2. It is noted that both the skin-friction coefficient and the wall couple stress coefficient decreases with an increase in $\operatorname{Pr}$ or $M$. Also, it is observed that both the skin friction and wall couple stress coefficient are increases with an increase in $G r$ or $R$ or $Q$ and $E c$.

Table 2: The values of $C_{f}$ and $C_{m}$ for different values of $G r, M, P r, R, Q$ and $E c$.

\begin{tabular}{|c|c|c|c|c|c|c|c|}
\hline$G r$ & $M$ & $P r$ & $R$ & $Q$ & $E c$ & $C_{f}$ & $C_{m}$ \\
\hline 1.0 & 1.0 & 0.71 & 1.0 & 0.1 & 0.01 & 4.49588 & 1.15936 \\
\hline 2.0 & 1.0 & & & & & 7.23624 & 1.86599 \\
\hline 4.0 & 1.0 & & & & & 13.68210 & 3.52796 \\
\hline 0.1 & 2.0 & & & & & 4.27547 & 1.10273 \\
\hline \multirow[t]{9}{*}{0.1} & 4.0 & & & & & 4.21725 & 1.08765 \\
\hline & 1.0 & 2.0 & & & & 2.59359 & 0.66883 \\
\hline & & 5.0 & & & & 2.03541 & 0.52488 \\
\hline & & 0.71 & 1.5 & & & 5.02602 & 1.29606 \\
\hline & & & 2.0 & 0.1 & & 5.31058 & 1.39556 \\
\hline & & & 1.0 & 0.0 & & 3.87953 & 1.00045 \\
\hline & & & & -0.1 & 0.01 & 3.44608 & 0.90959 \\
\hline & & & & 0.1 & 0.03 & 4.51326 & 1.18188 \\
\hline & & & & & 0.05 & 4.54989 & 1.20629 \\
\hline
\end{tabular}




\section{Conclusions}

An analysis is made to study the effects of an unsteady MHD flow of a micropolar fluid past a vertical porous plate in the presence of a thermal radiation and viscous dissipation with variable heat flux and heat generation/absorption. The following conclusions can be drawn:

- Fluid temperature increases as the heat is induced in the fluid or heat gets absorbed from the fluid to the surface.

- Increase in the temperature of the fluid as the viscosity dissipation parameter increases.

- The translational velocity across the boundary layer decreases with increasing values of $M$ and $P r$, while it increases with increasing values of $G r, K, \beta, R, E c$ and $Q$.

- The magnitude of micro-rotation decreases with the increasing values of $G r$ near the wall and $K$, while it increases with the increasing values of $M$ and $\beta$.

\section{References}

Abdel-Rahman, Saad, A. and Abdel-Wahed, M.S. (2011): Heat transfer over an unsteady moving surface with heat generation and thermal radiation in micropolar fluid in the presence of suction/injection, International Journal of Energy \& Technology, Vol.3, No.31, pp.1-7.

Ariman, T., Turk, M.A. and Sylvester, N.D. (1974): Review article-applications of micro-continuum fluid mechanics, International Journal of Engineering Science, Vol.12, pp. 273-293. http://dx.doi.org/10.1016/0020$\underline{7225(74) 90059-7 .}$.

Duwairi, H.M. (2005): Viscous and Joule Heating Effects on Forced Convection Flow from Radiate Isothermal Porous Surfaces, International Journal of Numerical Methods for Heat and Fluid Flow, Vol.15. No. 5, pp. 429440. http://dx.doi.org/10.1108/09615530510593620

Eringen, A.C. (1966): Theory of micropolar fluids, Journal of Mathematics and Mechanics, Vol.16, pp.1-18.

Eringen, A.C. (1972): Theory of thermo-micro fluids, Journal of Mathematical Analysis and Applications, Vol.38 pp. 480-496. http://dx.doi.org/10.1016/0022-247X(72)90106-0

El-Hakiem, M.A., Mohammadein, A.A., El-Kabeir, S.M.M., and Gorla, R.S.R. (1999): Joule heating effects on magnetohydrodynamic free convection flow of a micropolar fluid, International Communications in Heat and Mass Transfer, Vol. 26, No. 2, pp. 219-227. http://dx.doi.org/10.1016/S0735-1933(99)00008-1

Foraboschi ,F.P., Federico, I.D. (1964): Heat transfer in a laminar flow of non- Newtonian heat generating fluids, International Journal of Heat Mass Transfer,Vol.7, pp. 315-318. http://dx.doi.org/10.1016/00179310(64)90107-3

Gebhart, B. and Mollendorf, J. (1969): Viscous dissipation in external natural convection flows, Journal of Fluid Mechanics, Vol.38, pp.97-107. http://dx.doi.org/10.1017/S0022112069000061

Gnaneswara Reddy, M. (2012): Magnetohydrodynamics and radiation effects on unsteady convection flow of micropolar fluid past a vertical porous plate with variable wall heat flux, ISRN Thermodynamics, Vol. 2012, Article ID 146263, 8 pages. http://dx.doi.org/10.5402/2012/146263

Gorla, R.S.R. and Tornabene, R. (1988): Free convection from a vertical plate with non-uniform surface heat flux and embedded in a porous medium, Transport Porous Media,Vol.3,pp.95-106. http://dx.doi.org/10.1007/BF00222688.

Ibrahim, F.S., Elaiw, A.M. and Bakr, A.A. (2008): Influence of viscous dissipation and radiation on unsteady MHD mixed convection flow of micropolar fluids, Applied Mathematics \& Information Sciences, Vol.2, No.2, pp.143-162.

Ishak, A., Nazar, R., and Pop, I. (2006): Flow of a micropolar fluid on a continuous moving surface in the presence of viscous dissipation, Archive Mechanics, Vol.58, No. 6, pp.529-541.

Israel-Cookey, C., Ogulu, A. and Omubo-Pepple, V.B. (2003): Influence of Viscous dissipation on unsteady MHD free-convection flow past an infinite heated vertical plate in porous medium with time-dependent suction, International Journal of Heat Mass Transfer, Vol.46, pp.2305-2311. http://dx.doi.org/10.1016/S00179310(02)00544-6. 
Jena, S.K. and Mathur, M.N. (1981): Similarity solutions for laminar free convection flow of a thermomicropolar fluid past a non-isothermal vertical flat plate, International Journal of Engineering and Science,Vol.19, pp. 1431-1439.

Khedr, M.E.M., Chamkha, A.J., and Bayomi, M. (2009): MHD flow of a micropolar fluid past a stretched permeable surface with heat generation or a absorption,Nonlinear Analysis Modelling and Control, Vol.14, No. 1,pp. 27-40.

Kim, Y.J. (2000): Unsteady MHD convective heat transfer past a semi-infinite vertical porous moving plate with variable suction, International Journal of Engineering Science,Vol.38, pp.833-845.

http://dx.doi.org/10.1016/S0020-7225(99)00063-4.

Kim, Y.J. (2001a): Unsteady convection flow of micropolar fluids past a vertical porous plate embedded in a porous medium, Acta Mechanica,Vol.148,No.1-4, pp.105-116. http://dx.doi.org/10.1007/BF01183672

Kim, Y.J. (2001b): Unsteady MHD convection flow of polar fluids past a vertical moving porous plate in a porous medium, International Journal of Heat and Mass Transfer, Vol. 44, No. 15, pp. 2791-2799.

http://dx.doi.org/10.1016/S0017-9310(00)00332-X.

Mostafa, A.A., Mahmoud and Shimaa E. Waheed (2012): MHD stagnation point flow of a micropolar fluid towards a moving surface with radiation, Meccanica,Vol.47, pp.1119-1130. http://dx.doi.org/10.1007/s 11012011-9498-X.

Mohamed, R.A, Rida, S.Z., Arfa, A.A.M. and Said, M.(2011): Heat and mass transfer analysis on the flow of non-Newtonian micropolar fluid with uniform suction/blowing, heat generation, chemical reaction and thermophoresis effects, Proceeding of 2nd International Conference on Mathematics and Information Sciences, 9-13, Sohag, Egypt.

Okedoye, A. M. (2014): Unsteady MHD mixed convection flow past an oscillating plate with heat source/sink, Journal of Naval Architecture and Marine Engineering, Vol.11, pp.167-176.

http://dx.doi.org//10.3329/jname.v11i2.6477 .

Patowary, G. (2012): Effect of variable viscosity and thermal conductivity of micropolar fluid in a porous channel in presence of magnetic field, International Journal for Basic Sciences and Social Sciences (IJBSS), Vol.1, No.3, pp. 69-77.

Poornima, T., Sreenivasulu, P., and Bhaskar Reddy, N., (2014): Slip flow of casson rheological fluid under variable thermal conductivity with radiation effects, Heat Transfer-Asian Research, Vol.00, No.00, 2014.

http://dx.doi.org/10.1002/htj.21145

Rahman, M.M. and Sattar, M.A. (2006): Magnetohydrodynamic convective flow of a micropolar fluid past a continuously moving vertical porous plate in the presence of heat generation or absorption, Journal of Heat Transfer, Vol. 128, No. 2, pp.142-152. http://dx.doi.org/10.1115/1.2136918.

Raptis, A. (1998): Flow of a micropolar fluid past a continuously moving plate by the presence of rad iation, International Journal Heat Mass Transfer, Vol.41, pp.2865-2866. http://dx.doi.org/10.1016/S00179310(98)00006-4.

Rahman, M.M. and Sultana, T., (2008): Radiative heat transfer flow of micropolar fluid with variable heat flux in a porous medium, Nonlinear Analysis: Modelling and Control, Vol. 13, No. 1, pp.71-87.

Reddy, G.V.R., Ibrahim, S.M. and Bhagavan, V.S. (2014): Similarity transformations of heat and mass transfer effects on steady MHD free convection dissipative fluid flow past an inclined porous surface with chemical reaction, Journal of Naval Architecture \& Marine Engineering,Vol.11, pp.157-166. http://dx.doi.org/10.3329/jname.v 11i2.18313

Reddy, P. B. A. (2016): MHD boundary layer slip flow of a Casson fluid over an exponentially stretching surface in the presence of thermal radiation and chemical reaction, Journal of Naval Architecture and Marine Engineering, Vol.13,No.2016,pp.165-177. http://dx.doi.org/10.3329/jname.v13i2.23537

Sharma, R.C. and Gupta, U. (1995): Thermal convection in micropolar fluids in porous medium, International Journal of Engineering Science, Vol. 33, No. 13, pp.1887-1892. http://dx.doi.org/10.1016/0020-7225(95)00047$\underline{2}$

Soundalgekar, V.M. (1972): Viscous dissipation effects on unsteady free convective flow past an infinite vertical porous plate with constant suction, International Journal Heat Mass transfer, Vol.15, pp.1253-1261.

http://dx.doi.org/ 10.1016/0017-9310(72)90189-5 
Soundalgekar, V.M. (1973): Free convection effects on the oscillatory flow past an infinite, vertical, porous plate with constant suction, Proceedings of Royal Society, London A,Vol.333, pp.25-36.

http://dx.doi.org/10.1098/rspa.1973.0046

Soundalgekar, V.M. (1977): Free convection effects on Stokes problem for a vertical plate, ASME Journal of Heat Transfer, Vol.99, pp.499-501. http://dx.doi.org/10.1016/0029-5493(79)90127-4.

Sreenivasulu, P., Poornima, T., Bhaskar Reddy, N.(2017): Variable thermal conductivity influence on hydromagnetic flow past a stretching cylinder in a thermally stratified medium with heat source/sink, Frontiers in Heat and Mass Transfer (FHMT),Vol. 9, No.20, pp.1-7. http://dx.doi.org/10.5098/hmt.9.20

Sreenivasulu, P., Poornima, T. and Bhaskar Reddy, N.(2014): Variable Suction Effect on Magnetohydrodynamic Convective and Radiating Flow past a Vertical Porous Moving Surface, World Applied Sciences Journal ,Vol.32,No.8,pp. 1484-1491. http://dx.doi.org/10.5829/idosi.wasj.2014.32.08.1159

Sultana, M., Haque, M.M., Alam, M.M., Ferdows, M. and Postelnicu, A. (2011): Micropolar fluid behavior on MHD heat transfer flow through a porous medium with induced magnetic field by finite difference method, European Journal of Scientific Research,Vol.53 No.3, pp.477-490. 